\title{
Modelling the effects of increased physical activity on coronary heart disease in England and Wales
}

\author{
Bhash Naidoo, Margaret Thorogood, Klim McPherson, Louise J Gunning-Schepers
}

\begin{abstract}
Objective - To investigate the use of computer models as tools for policy makers in evaluating physical activity interventions aimed at reducing deaths from coronary heart disease (CHD).

Design - The cell-based computer model Prevent, adapted to simulate risk factor interventions for an English and Welsh population, was used to simulate the effect of two strategies for increasing physical activity levels in respect of CHD mortality over 25 years. The first strategy involved a $25 \%$ increase in the proportion of $15-64$ year olds who were moderately active, while the second strategy involved a similar increase in the proportion who were vigorously active. The effects of focusing on narrower age ranges and on people at different initial activity levels were also explored.
\end{abstract}

Main results - The simulations showed a small reduction in the CHD death rates less than $0.15 \%$ and $0.06 \%$ for men and women respectively. The strategies would postpone up to 12100 deaths over 25 years, comparable to the effect of a $2 \%$ reduction in smoking prevalence. The strategies seemed as if they would be more effective if they concentrated on men rather than women, on those over 45 years of age as opposed to all or younger age groups, and on the least active members of the population rather than those already taking some exercise.

Conclusion - The use of computer modelling for simulating physical activity strategies has shown that concentrating these interventions on older sedentary men will produce the greatest health gain, but efforts to encourage smoking cessation may be more effective in terms of years of life saved.

( $($ Epidemiol Community Health 1997;51:144-150)

It is often difficult to evaluate the effectiveness of health promotion interventions by experimental or observational methods since the time scales necessary are too long. An interim solution is to use computer models which will simulate the effects of different interventions within a population, using available data on risk factor prevalence and the attributable risks of disease mortality, and projecting the results over several generations. Such models are po- tentially useful in assessing and comparing the relative health gain of alternative intervention strategies. This is a new methodology, and this work should be seen as an early step in attempting to explore the application of these techniques.

Lack of physical activity has been shown to be a strong independent risk factor ${ }^{1}$ for death from coronary heart disease (CHD). In a metaanalysis, Berlin and Colditz ${ }^{2}$ calculated a 1.9 fold increased relative risk for CHD mortality associated with a sedentary lifestyle compared with a vigorously active lifestyle. Population surveys have shown that only a small percentage of the British population take enough exercise to protect against CHD. ${ }^{3}$ Inactivity, then, may be an important cause of CHD mortality. The promotion of physical activity is currently the focus of discussion. ${ }^{4}$ The number of "prescription for exercise" schemes, in which general practice patients are given free or reduced entrance to exercise facilities, is growing rapidly.

The Government's white paper The Health of the Nation ${ }^{5}$ in 1992 introduced targets for the reduction in the death rates for CHD. It is not clear how the promotion of physical activity could most effectively contribute to these targets. To contribute to the debate we have modelled the effects of increasing activity in the population, looking at the effects of targeting differing exercise levels, age and gender groups.

This work was undertaken as part of the health gain project, funded jointly by the Health Education Authority and North Thames Regional Health Authority, which aims to provide policy makers with predictions of the effects of changing resource priorities, simulating the effect of shifts in resource allocation using computer modelling.

\section{Methods}

CURRENT ACTIVITY LEVELS

Table 1 has been adapted from the Allied Dunbar national fitness survey (ADNFS), and shows the proportion of men and women in England and Wales by age group who are active at different levels. Activity levels were based on 20 minute periods of physical activity in a four week period, and defined as:

Vigorous - 12 or more occasions of activities at $7.5 \mathrm{kcal} / \mathrm{minute}$ and above, such as squash, running, football, swimming, tennis, aerobics, and cycling (if out of breath or sweaty).

Moderate - 12 or more occasions of activities between 5 and $7.5 \mathrm{kcal} /$ minute, such as foot- 
Table 1 Prevalence of physical activity by sex, age group, and exercise level

\begin{tabular}{lrrrrrr}
\hline \multicolumn{7}{l}{ Age groups (\%) } \\
\cline { 2 - 7 } Exercise level & $16-24$ & $25-34$ & $35-44$ & $45-54$ & $55-64$ & $65+$ \\
\hline Men: & & & & & & \\
$\quad$ Vigorous & 28.9 & 20.4 & 12.8 & 8.4 & 4.1 & 1.4 \\
$\quad$ Moderate & 37.7 & 41.6 & 40.8 & 38.9 & 29.8 & 19.6 \\
$\quad$ Light & 27.8 & 30.1 & 36.5 & 38.1 & 36.5 & 35.9 \\
$\quad$ Sedentary & 5.6 & 7.9 & 9.9 & 14.6 & 29.6 & 43.1 \\
Women: & & & & & & \\
$\quad$ Vigorous & 9.7 & 7.6 & 4.1 & 2.9 & 1.8 & 0.2 \\
$\quad$ Moderate & 37.1 & 46.5 & 46.0 & 37.3 & 31.8 & 19.5 \\
Light & 44.5 & 39.4 & 40.0 & 46.3 & 45.3 & 40.3 \\
Sedentary & 8.7 & 6.5 & 9.9 & 13.5 & 21.1 & 40.0 \\
\hline
\end{tabular}

ball, swimming, tennis, aerobics and cycling (if not out of breath or sweaty), table tennis, golf, social dancing and exercises (if out of breath or sweaty).

Light - one to 11 occasions of activities at $5 \mathrm{kcal} / \mathrm{minute}$ and above, such as table tennis, golf, social dancing and exercises (if not out of breath or sweaty), bowls, fishing, darts and snooker.

Sedentary - no occasions of activities above $5 \mathrm{kcal} / \mathrm{minute}$.

\section{THE STRATEGIES}

To evaluate the potential effect of different strategies for physical activity promotion we explored two options representing interventions targeted at sedentary, lightly active, and moderately active people. These strategies were as follows:

Strategy 1 - to encourage those who are either sedentary or lightly active to undertake moderate activity, thus increasing by $25 \%$ the proportion of the population aged between 15-64 which is moderately active.

Strategy 2 - to encourage those who are either sedentary, lightly active or moderately active to undertake vigorous activity, thus increasing by $25 \%$ the proportion of the population aged between 15 and 64 which is vigorously active.

We have investigated separately the effect of these strategies in men and in women, and in different age groups.

\section{"PREVENT"}

Prevent ${ }^{6}$ was developed by one of us (LJ Gunning-Schepers) in 1988. It is a cell-based simulation model that can estimate the health benefits for a population of changes in risk factor prevalence due to trends and interventions over a maximum period of 50 years, both in terms of proportional changes in disease specific incidence and in terms of absolute changes in such parameters as disease specific and total mortality.

After the user has specified the risk factor to be modified, the model first calculates the autonomous development of risk factor prevalences due to existing trends. Then the user specifies change in risk factor prevalences after the intervention and the model calculates the development due to the intervention and these trends. Next the model calculates the autonomous development of all other risk factors that share diseases with the intervention risk factors. Finally the results of the calculations are applied to two populations - one with only the autonomous developments and the other with both the autonomous developments and the intervention effects.

The differences between the two populations are attributed to the intervention, with the output given in terms of total and disease specific mortality (details of the calculations are presented in Appendix 1). It was originally produced to simulate a Dutch population with base year 1985, but we have adapted it to simulate an English and Welsh population with base year 1991 .

We have used Prevent to simulate proposed changes for the English and Welsh population, modelling the hypotheses of a graded effect for the health benefits of physical activity, using high risk and population strategies to achieve the set strategies.

\section{ADAPTING PREVENT}

Prevent does not normally include lack of physical activity as a risk factor, therefore the following data were input for the England and Wales population:

- Physical activity level categories: sedentary, light, moderate, and vigorous,

- Prevalence of physical activity by sex, age groups and activity level,

- Relative risk of CHD death due to lack of physical activity by sex, age groups, and activity level,

- Two time intervals, the first, LAT, giving the time between taking up physical activity and a person's relative risk begins to decrease, and the second, LAG, giving the time between a person's relative risk beginning to decrease and when it reaches its lowest value for the new level of physical activity,

- The remnant relative risk, which is the lowest possible relative risk that an ex-exposed person has after LAT + LAG time has elapsed on taking up a new level of physical activity.

\section{LACK OF PHYSICAL ACTIVITY AND CHD DEATH RISK}

The way in which lack of physical activity affects CHD mortality is not fully understood. Although it has been shown to be an independent risk factor; the actual mechanism by which risk of CHD death decreases with increased levels of physical activity is not clear. We used the hypothesis that there is an inverse and graded relationship between CHD risk and physical activity, as proposed by Shaper. ${ }^{7}$

\section{RELATIVE RISKS}

The relative risks for $\mathrm{CHD}$ mortality due to lack of physical activity were taken from a comprehensive meta-analysis. ${ }^{2}$ No relative risk for light activity was produced in the metaanalysis, so we derived a hypothetical relative 
Table 2 Relative risk (RR) of cornary heart disease mortality in relation to exercise level

\begin{tabular}{ll}
\hline Exercise levels & $R R$ \\
\hline Vigorous & 1.0 \\
Moderate & 1.4 \\
Light & $1.7^{*}$ \\
Sedentary & 1.9 \\
\hline${ }^{*}$ From interpolation &
\end{tabular}

* From interpolation.

risk by interpolation. The relative risks are shown in table 2 .

These relative risks are unfortunately not known separately by age or sex, and moreover the definition of exercise levels is not completely clear with respect to duration and calorific output. We have assumed that these levels are similar to those used in the ADNFS data.

The problems of "fitting" available prevalence and relative risk data to the English and Welsh population, and then tailoring these for input into Prevent were considerable and entailed making compromises. Prevent was not designed to calculate the effect of members of one risk factor exposure group moving into a number of different exposure groups; it was designed solely for modelling movement from an exposed to a non-exposed category. The remnant risks in the model had to be adapted to permit movement between different levels of exposure. An additional problem was that only age group divisions beginning with multiples of five are permitted, so the youngest age for intervention had to be 15 rather than 16 years.

\section{MODEL ASSUMPTIONS}

In setting up Prevent we have to set certain calculation options and variables, and these translate into a number of assumptions about the way in which an intervention would affect physical activity in the population and the process by which physical activity influences $\mathrm{CHD}$ mortality.

We assumed that the intervention started in 1994 and continued for the next 11 years, with the prevalences of the strategies being achieved in 2005, a target year for the Health of the Nation. ${ }^{3}$ The population was simulated for a further 14 years after the end of the intervention.

The mechanism by which physical activity affects CHD mortality is essentially described by the time periods over which a person's risk decreases and the risk it declines to. Within our model we have assumed that on taking up exercise a person's relative risk begins to decrease immediately, and that one year after taking up a new level of exercise a person's relative risk decreases to that of people exercising at that level. We have performed some sensitivity analysis by increasing these time periods when running the model.

In terms of the remnant risk we have assumed that a person's previously less active lifestyle will not continue to have a detrimental effect on their health, and that they will take on the relative risk attributed to their current physical activity level.
As our main interest was to investigate how changing a population's physical activity levels might affect its CHD mortality, we chose to assume that physical activity decreased the risk of CHD death only and did not affect other diseases. In addition, we assumed that physical activity did not affect the other risk factors that Prevent includes, such as hypertension, cholesterol, and obesity.

We assumed that the proposed interventions would change an age group's behaviour for the entire simulation period ( 25 years), so that as cohorts age during the simulation period and move from one age group to the next, some people will take up a new physical activity level which may be at a lower or higher level than the level they achieved in their previous age group.

We have also assumed that any changes in the prevalence of physical activity within the population would be solely as a result of the interventions, and that there would be no background risk factor trends in the population that is, without an intervention there would be no change over time in rates of physical activity.

Care needs to be taken in choosing either the age group or the cohort option for the calculations in Prevent, since the two options can give markedly different results. The age group option should be used when considering a risk factor that is predominately age dependent, such as hypertension. An intervention that causes a behavioural change, such as cigarette smoking cessation, is more likely to affect a birth cohort which retains the change as it ages. We chose to model physical activity as having an age group effect, although we also tested the model with the cohort option to see how this affected the results.

Two scenarios were used to model each strategy to provide lower and upper limits of estimated outcomes. For strategy 1 , which aimed to increase the percentage of the population with a moderate level of physical activity, we simulated two scenarios that targeted either the sedentary group, or those already undertaking light activity. Strategy 2, which aimed to increase the number of people undertaking

Table 3 Coronary heart disease mortality per 100000 for men and for women aged under 95 achieving each strategy

\begin{tabular}{|c|c|c|c|c|c|}
\hline & \multirow[b]{2}{*}{ Year } & \multicolumn{2}{|c|}{ Strategy 1} & \multicolumn{2}{|c|}{ Strategy 2} \\
\hline & & Lower & Upper & Lower & Upper \\
\hline \multicolumn{6}{|l|}{ Men: } \\
\hline & $\begin{array}{l}1994 \\
2019\end{array}$ & $\begin{array}{l}1382.5 \\
1381.3\end{array}$ & $\begin{array}{l}1382.5 \\
1380.5\end{array}$ & $\begin{array}{l}1382.5 \\
1382.1\end{array}$ & $\begin{array}{l}1382.5 \\
1381.6\end{array}$ \\
\hline \multicolumn{6}{|l|}{ Women: } \\
\hline & $\begin{array}{l}1994 \\
2019\end{array}$ & $\begin{array}{l}915 \\
914.7\end{array}$ & $\begin{array}{l}915 \\
914.5\end{array}$ & $\begin{array}{l}915 \\
914.9\end{array}$ & $\begin{array}{l}915 \\
914.8\end{array}$ \\
\hline
\end{tabular}

Table 4 Modified relative risk of coronary heart disease mortality in relation to exercise level and age group

\begin{tabular}{lllll}
\hline \multirow{5}{*}{ Exercise levels } & \multicolumn{2}{l}{ Age groups } & \multicolumn{3}{l}{} \\
\cline { 2 - 5 } & $15-44$ & $45-54$ & $55-64$ & $65+$ \\
\hline Vigorous & 1.0 & 1.0 & 1.0 & 1.0 \\
Moderate & 1.4 & 1.3 & 1.3 & 1.3 \\
Light & 1.7 & 1.6 & 1.5 & 1.4 \\
Sedentary & 1.9 & 1.8 & 1.7 & 1.6 \\
\hline
\end{tabular}


Table 5 Example calculation of potential impact factor (PIF) for the risk factor, lack of physical activity, in men aged 55-64 under strategy 1, using the lower limit of the intervention

\begin{tabular}{llllll}
\hline $\begin{array}{l}\text { Exercise } \\
\text { level }\end{array}$ & $\begin{array}{l}\text { Original } \\
\text { prevalence }\end{array}$ & $\begin{array}{l}\text { Intervention } \\
\text { prevalence (1) }\end{array}$ & $\begin{array}{l}\text { Relative } \\
\text { risk }\end{array}$ & & \\
\hline Vigorous & 0.041 & 0.041 & 1 & PIDR(0) & 1.6411 \\
Moderate & 0.298 & 0.391 & 1.4 & PIDR(1) & 1.6132 \\
Light & 0.365 & 0.272 & 1.7 & & \\
Sedentary & 0.296 & 0.296 & 1.9 & PIF & 0.017 \\
Sum & 1 & 1 & & & \\
\hline
\end{tabular}

PIDR = proportion incidence density ratio.

vigorous activity, was simulated using two scenarios which assumed that sedentary people would increase their activity to this level or that those already taking moderate exercise would increase their level.

\section{INTERVENTION OUTPUTS}

The outputs from the simulated interventions have been produced in terms of:

- Percentage reduction in CHD mortality rate (compared with 1994),

- Actual years of life gained.

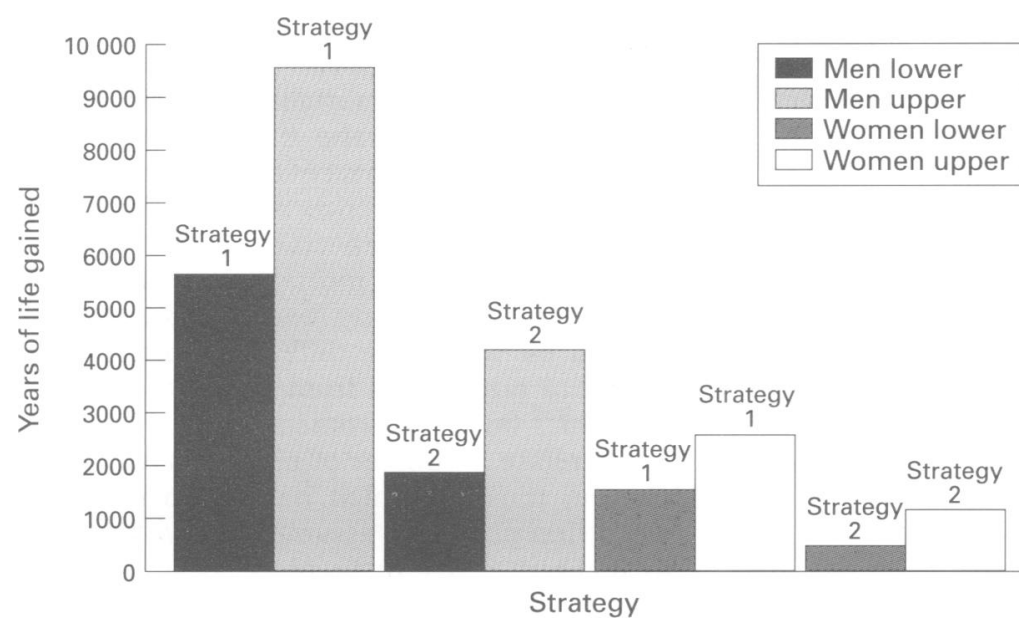

Figure 1 Actual years of life gained for men and for women aged under 95 years and achieving each intervention strategy by the year 2019.

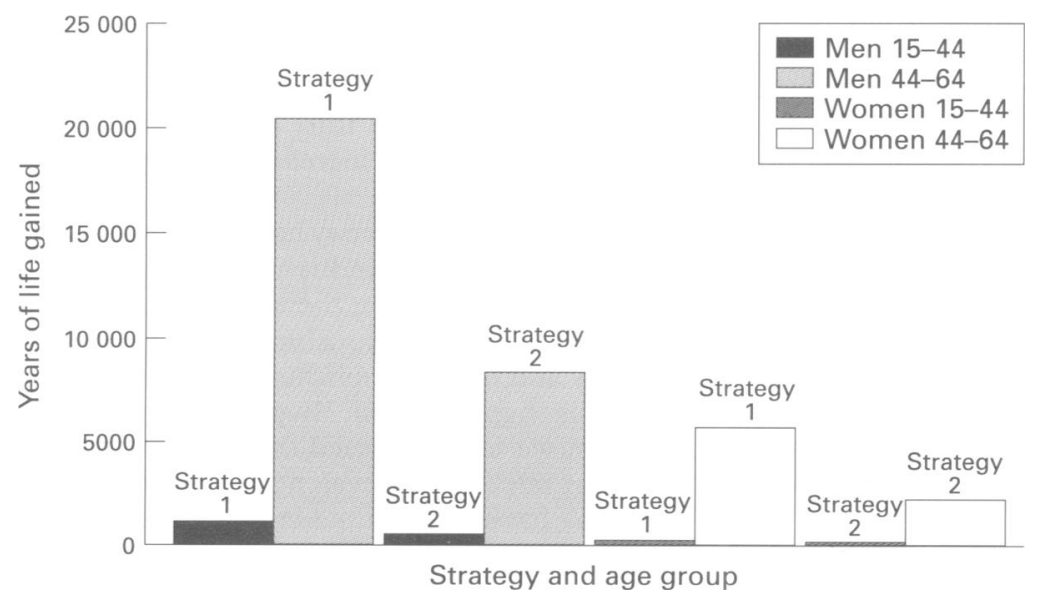

Figure 2 Actual years of life gained for men and women aged under 95 years and achieving each intervention strategy by the year 2019, targeting by age groups.

\section{Results}

There would be a very small reduction in the death rate from CHD associated with achievement of each of the two strategies in men and women under 95 years of age, see table 3 . This represents a fall of between $0.03 \%$ and $0.15 \%$ in men and between $0.01 \%$ and $0.06 \%$ in women, across all age groups combined.

While the proportional reduction in the death rate appears disappointing, the actual years of life gained by the year 2019 are not insubstantial (fig 1). The time trajectory is roughly linear from 1994, showing that the interventions are delaying deaths, although not enough to affect the mortality rate greatly. There are similar trends for both men and women, although the number of deaths postponed for men is about four times greater than for women. For both the upper and lower estimates strategy 1 is the most effective in achieving life years gained. (Appendix 2, tables 6,7 , and 8 gives the data for the figures).

To examine the differing effect of targeting exercise interventions at various age groups, we modelled the life years gained for each of the two strategies making the assumptions that the strategy would concentrate on the older age groups (45-64 years of age) or on the younger age groups (15-44 years of age). Figure 2 shows this comparison in men and women in terms of actual years of life gained, using the mean of the two scenarios simulated. For both men and women, the greatest gain can be achieved by concentrating on the older group. Again strategy 1 was the most effective and, as before, there is less gain achievable in women than in men.

\section{SENSITIVITY ANALYSIS}

It is possible that the increased relative risk associated with inactivity is attenuated in older people. If this is true then the model will overestimate the effect of interventions in the elderly. We therefore carried out some sensitivity analysis for the simulations targeting those people aged 45-64 years of age, in which they were given lower relative risks for each level of physical activity (See table 4). This change made only a small difference to the number of deaths postponed, and targeting the older age groups still produced the greatest benefit (figs 3 and 4). Modifying the remnant relative risks did not affect the results for those aged 15-44 years old.

Age group interventions were also simulated, using the cohort option as well as the modified relative risks, to check if this improved the results for those aged 15-44 years old. Under this option the intervention would be responsible for changing people's behaviour for the entire simulation period, in that those people that take up a new physical activity level will continue with this level of activity for 25 years and not revert to a lower level of physical activity. Figures 3 and 4 show that this increased the actual years of life gained when targeting the younger age group, but concentrating on people 45-64 years old still achieved the most health gain. 


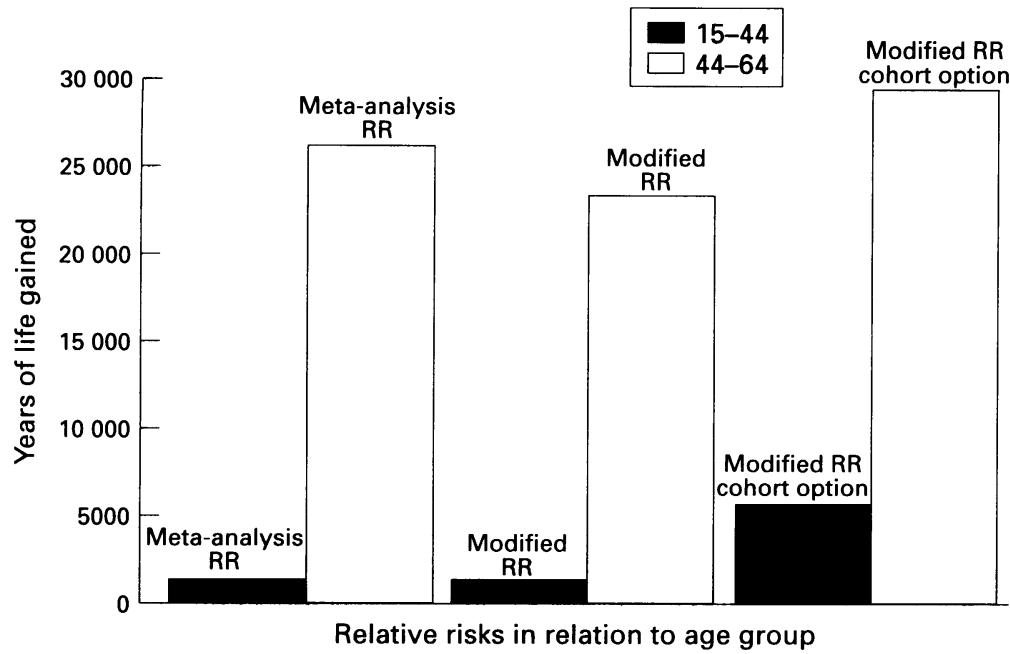

Figure 3 Actual years of life gained for men and women aged under 95 years and achieving strategy 1 by the year 2019, targeting by age groups using modified relative risks and the cohort option.

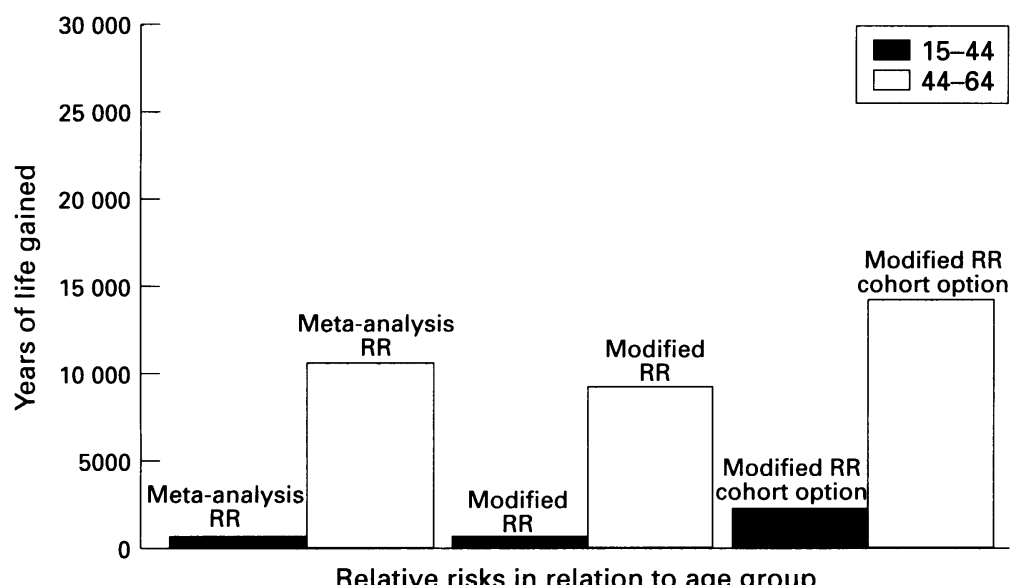

Figure 4 Actual years of life gained for men and for women aged under 95 years and achieving strategy 2 by the year 2019, targeting by age groups using modified relative risks and the cohort option. in those over the age of 65 , while these interventions concentrate on those under 65 . The most effective strategy would achieve a $2.6 \%$ reduction for men and a $2 \%$ reduction for women in CHD mortality up to 65 years.

The greater effect of strategy 1 as compared with strategy 2 could be because more people receive the intervention, since $39.3 \%$ of the population aged between 15 and 64 are moderately active, while only $10.7 \%$ are vigorously active. Increasing each category by $25 \%$ results in $49.1 \%$ of the population in this age group undertaking moderate activity and $13.4 \%$ undertaking vigorous activity.

The proportion of the population at risk of CHD in the older age group has a significant impact on the relative effectiveness of the interventions by age group. This is emphasised when using attenuated relative risks for the older age group in the simulation, since the actual years of life gained for this age group still outweighs that for the younger age group, even though the younger age group has higher relative risks.

The use of the cohort option in the simulation showed that the targeting of older age groups was still more effective, in terms of the actual years of life gained over 25 years, than targeting younger cohorts who would retain their new physical activity levels over time. This again may be an implication of the influence of the higher CHD mortality rates among the older age groups in the England and Wales population on the impact of these interventions.

The marked difference between the results for men and women reflects the fact that women have a much lower mortality rate for CHD than men, particularly between the ages of 25 and 64 where the difference in the CHD mortality rate ranges from three to six and a half times lower for women.

There are a number of problems with using Prevent, most of which have been discussed when describing the assumptions we have had to make in order to fit physical activity data into the model. These include the fact that the model was initially designed for shifting exposure groups to non-exposure groups and having to use multiples of five for the age group divisions. One of the major drawbacks has been the lack of any estimate of changes in morbidity, especially if physical activity has much bigger effects on the prevalence of non-fatal disease. Work is currently underway to develop a new version, ${ }^{9}$ which takes into account morbidity, and this will greatly increase the applicability of the model.

The Prevent model may have underestimated the gain in mortality reduction from increasing physical activity. Since there is no clear understanding of the relationship between physical activity and other established CHD risk factors such as hypertension, hyperlipidaemia, and obesity, we have assumed that physical activity will not affect the prevalence of these risk factors. We have also assumed that increased physical activity will not affect mortality from any other cause of death included in the Prevent model. These are cerebrovascular accident, chronic obstructive lung disease, lung cancer, 


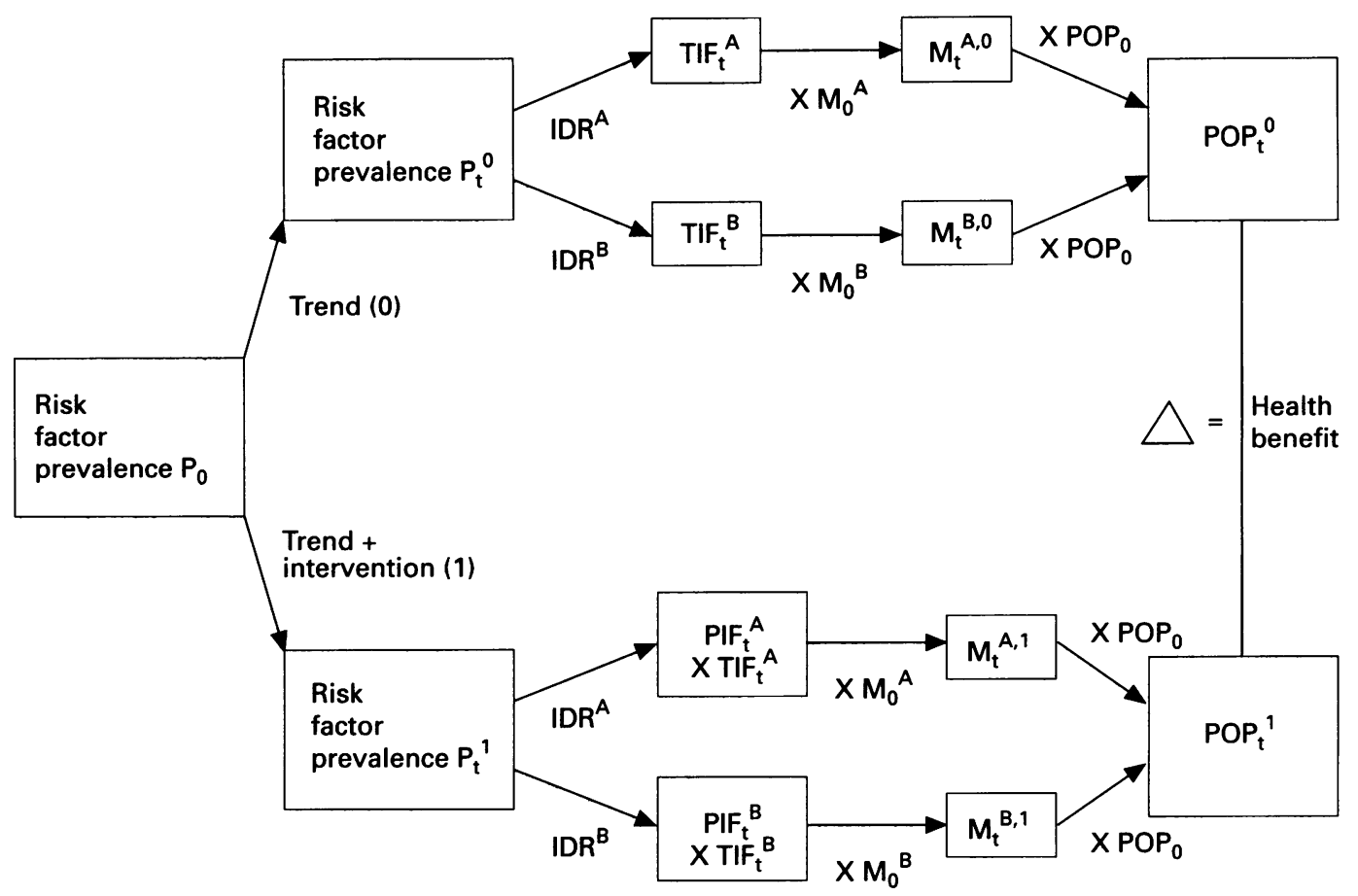

Figure 5 The basic version of the Prevent model.

cirrhosis of the liver, breast cancer, traffic accidents, and accidental falls.

Even if Prevent has underestimated the total gain possible from increasing physical activity, the relative gain of different strategies carries an important message. Our work with Prevent indicates that the greatest health gain can be achieved by concentrating on sedentary people, on older people, and on men. This may seem a contradiction of Rose's ${ }^{10}$ argument that preventive strategies which concentrate on a minority at high risk produce less health gain than strategies which intervene across the whole population, since the minority at high risk contribute a small proportion of adverse events. However, the majority of older men ( 45 plus) do not undertake even moderate exercise, and older men account for about $67 \%$ of coronary death under the age of 75 . In this case, then, the most effective strategy is the one which concentrates on that section of the population which is contributing the majority of deaths.

1 Powell KE, Thompson PD, Caspersen CJ, Kendrick JS. Physical activity and the incidence of coronary heart disease. Annu Rev Public Health 1987;8:253-87.

2 Berlin JA, Colditz GA. A meta-analysis of physical activity in the prevention of coronary heart disease. Am $\mathcal{F}$ Epidemiol 1990;132:612-27.

3 Sports Council and Health Education Authority. Allied Dunbar national fitness survey. London: Sports Council, Department of Health, 1992.

4 Health Education Authority. Moving on - International perspectives on promoting physical activity. London: HEA, 1994.

Sectretary of State for Health. The Health of the Nation. London: HMSO, 1992.

6 Gunning-Schepers LJ. The health benefits of prevention, a Gunning-Schepers LJ. The health benefits of prevention
simulation approach. Health Policy 1989;12:1-256.

7 Shaper AJ, Wannamethee G. Physical activity and ischaemic heart disease in middle-aged British men. Br Heart $f 1991$; 66:384-94

8 Silagy CA, Fowler GH. Systematically reviewing the effectiveness of pharmacological and non-pharmacological smoking cessation methods. Fournal of Smoking-Related Diseases 1994;5 (Suppl. 1):295-303

9 Barendregt JJ. From Prevent to NIMPH and back: Some methodologic issues in public health modelling. Proceedings of the European Public Health Association Conference, December 1994, Copenhagen.

10 Rose G. The strategy of preventive medicine. Oxford: Oxford University Press, 1992.
Appendix 1

BASIC METHODOLOGY OF THE PREVENT MODEL ${ }^{6}$

Figure 5 illustrates the basic version of the Prevent model.

$$
\begin{gathered}
P I D R_{t}^{r, j, z, s, A}=\sum_{n=1}^{c n} \sum_{i=0}^{I D} P_{t-L A T T_{, s}, j, s}^{r, j, A} R^{r, z, s, A, n, i} \\
T I F_{t}^{r, z, s, A}=\frac{P I D R^{r, 0, z, s, A}-P I D R_{t}^{r, 0, z, s, A}}{P I D R_{0}^{r, 0, z, a, A}} \\
P I F_{t}^{r, z, s, A}=\frac{P I D R^{r, 0, z, s, A}-P I D R_{t}^{r, 1, z, s, A}}{P I D R_{t}^{r, 0, z, a, A}} \\
M_{t}^{0, s, A}=M^{s, A}-\sum_{z=1}^{z t} T I F_{t}^{z, s, A} M^{z, s, A} \\
M_{t}^{1, s, A}=M^{s, A}-\sum_{z=1}^{z t}\left[1-\left(T I F_{t}^{z, s, A}\right)\left(1-P I F_{t}^{z, s, A}\right)\right] M^{z, s, A}
\end{gathered}
$$

Where:

A: index for age.

t: index for time.

i: index for ex-exposure level.

P: proportion.

ID: total number of ex-exposure levels.

IDR: incidence density ratio.

PIF: potential impact fraction - the incidence that is avoided by a preventive intervention as a proportion of the incidence that would have occurred in that population without the intervention.

TIF: trend impact fraction - the incident cases prevented at a certain moment in time, by an autonomous change in risk factor prevalence, as a proportion of the incident cases that would have occurred at that time in the absence of change. LAT: the period between the end of exposure to the risk factor and the first effect on disease specific mortality. 
cn: total number of exposure categories.

$\mathrm{n}$ : index for exposure category.

$\mathrm{r}$ : index for risk factor.

$\mathrm{j}=0,1$ : index for trend (0) or intervention population (1).

s: index for sex.

$\mathrm{z}$ : index for disease.

$\mathrm{M}^{\mathrm{s}, \mathrm{A}}$ : constant overall mortality quotient.

$\mathbf{M}_{t}^{j, s, A}$ : adjusted overall mortality quotient.

$\mathrm{M}^{\mathrm{z}, \mathrm{s}, \mathrm{A}}$ : disease specific mortality quotient.

Table 6 Actual years of life gained (absolute numbers) for men and for women aged under 95 achieving each strategy by 2019

\begin{tabular}{llllll}
\hline & \multicolumn{2}{l}{ Strategy 1} & & \multicolumn{2}{l}{ Strategy 2 } \\
\cline { 2 - 3 } \cline { 5 - 5 } \cline { 5 - 5 } & Lower & Upper & & Lower & Upper \\
\hline Men & 5613 & 9537 & 1866 & 4167 \\
Women & 1511 & 2556 & 501 & 1129 \\
\hline
\end{tabular}

Table 5 exemplifies an intervention on one risk factor for one age group with no background trends for that risk factor. It shows that this intervention will result in a reduction of $1.7 \%$ in CHD mortality for men aged 55-64.

\section{Appendix 2}

Tables 6,7 , and 8 below give the data on which figures 2,3 , and 4 are based.

Table 7 Actual years of life gained (absolute numbers) for men and for women aged under 95 achieving each strategy by 2019, targeting by age groups

\begin{tabular}{llllll}
\hline & \multicolumn{3}{l}{ Strategy 1} & & \multicolumn{2}{l}{ Strategy 2 } \\
\cline { 2 - 3 } \cline { 6 - 6 } \cline { 5 - 6 } & $15-44$ & $45-64$ & & $15-44$ & $45-64$ \\
\hline Men & 968 & 20437 & & 401 & 8238 \\
Women & 155 & 5675 & 64 & 2265 \\
\hline
\end{tabular}

Table 8 Actual years of life gained (absolute numbers) for men and for women aged under 95 achieving strategy 1 and Strategy 2 by 2019, targeting by age groups using modified relative risks (RR) and using the cohort option

\begin{tabular}{|c|c|c|c|c|c|c|}
\hline & \multicolumn{2}{|l|}{ Total } & \multicolumn{2}{|c|}{ Modified $R R$} & \multicolumn{2}{|c|}{ Cohort modified $R R$} \\
\hline & $15-44$ & $45-64$ & $15-44$ & $45-64$ & $15-44$ & $45-64$ \\
\hline $\begin{array}{l}\text { Strategy } 1 \\
\text { Strategy } 2\end{array}$ & $\begin{array}{r}1123 \\
465\end{array}$ & $\begin{array}{l}26112 \\
10503\end{array}$ & $\begin{array}{r}1123 \\
465\end{array}$ & $\begin{array}{r}23283 \\
9104\end{array}$ & $\begin{array}{l}5573 \\
2130\end{array}$ & $\begin{array}{l}29484 \\
14321\end{array}$ \\
\hline
\end{tabular}

